\title{
Maternal knowledge, outcome expectancies and normative beliefs as determinants of cessation of exclusive breastfeeding: a cross-sectional study in rural Kenya
}

Constance A. Gewa ${ }^{1 *}$ and Joan Chepkemboi ${ }^{2}$

\begin{abstract}
Background: Despite the importance of multiple psychosocial factors on nutrition-related behavior, very few studies have explored beyond the role of mothers' knowledge and perception of child-focused outcomes on the duration of exclusive breastfeeding in Africa. Our objective was to determine the relationships among mothers' knowledge, outcome expectancies, normative beliefs, and cessation of exclusive breastfeeding in rural Kenya.

Methods: A cross-sectional survey was conducted among 400 mothers of children, 0-24 months old, in rural Kenya. Early child-feeding practices, knowledge of breastfeeding recommendations, beliefs associated with impact of exclusive breastfeeding on child- and mother-focused outcomes and perception of acceptability of exclusive breastfeeding by important others were examined. Cox regression analysis was used to assess the relationship between independent variables of interest and cessation of exclusive breastfeeding.

Results: Being knowledgeable of breastfeeding-related recommendations, positive beliefs on the impact of exclusive breastfeeding on child- focused outcomes, having a more positive perception of the impact of exclusive breastfeeding on mother-focused outcomes and a more positive perception of acceptability of exclusive breastfeeding by important others were associated with significantly lower risks of premature cessation of exclusive breastfeeding.

Conclusion: In addition to knowledge levels, mothers' beliefs play an important role in mothers' decisions to practice exclusive breastfeeding. Mother's beliefs on the impact of exclusive breastfeeding on the mother's health, physical appearance and ability to engage in other activities were shown to have the strongest relationship with premature cessation of exclusive breastfeeding. Addressing these beliefs has the potential to contribute to more effective exclusive breastfeeding promotion efforts in rural Kenya.
\end{abstract}

Keywords: Exclusive breastfeeding, Knowledge, Outcome expectancies, Normative beliefs, Rural Kenya

\footnotetext{
* Correspondence: cgewa@gmu.edu

'Department of Nutrition \& Food Studies, College of Health \& Human

Services, George Mason University, 4400 University Dr. MSN 1 F8, Fairfax, VA

22030, USA

Full list of author information is available at the end of the article
} 


\section{Background}

Exclusive breastfeeding (EBF) has the potential to significantly reduce infant mortality and protect against infection and disease. According to the World Health Organization (WHO), it is recommended that all children should be exclusively breastfed for the first 6 months of life [1, 2]. In 2008-2009, it was estimated that only $13 \%$ of children within the 4-5 month age group were exclusively breastfed in Kenya [3]. Only $2 \%$ of children in two slum areas of Nairobi and $8 \%$ of children in rural Kenya were exclusively breastfed for the first six months of life $[4,5]$. After remaining low for many years, EBF rates seem to be on the rise in Kenya. Results from the most recent demographic health survey showed that $61 \%$ of children less than six months of age were still exclusively breastfeeding, with $45 \%$ of children within the 4-5 month age group still exclusively breastfeeding at the time of the survey [6]. These rates, however, are still below the $80 \%$ target set for 2017 [7]. A large proportion of new mothers rely on health workers for breastfeeding-related guidance, which is often provided via government-sponsored and private-run maternal and child health clinics $(\mathrm{MCH})$ in the country. While increased knowledge of recommended breastfeedingrelated practices is important, we believe that an improved understanding of person- and environmentrelated factors is integral to providing more effective EBF promotion efforts in Kenya. Women's roles outside the home have continued to rise over the years. Such changes may influence a mother's perception of their role in infant and young child feeding vis-à-vis that of other family members and impact on their ability to practice EBF. Beliefs on whether important people approve or disapprove of EBF (hereon referred to as normative beliefs) and beliefs about the outcomes of EBF (hereon referred to as expected outcomes) are bound to influence mothers' attitudes towards and motivation to practice EBF within the first six months of their child's life. Expected outcomes can be loosely divided into two categories, namely health and personally meaningful outcomes [8]. Examples of EBF-related health outcomes may include child's growth, risk of childhood illnesses such as diarrhea and mother's weight. EBF-related personally meaningful outcomes vary and may include convenience, mother's energy levels and personal appearance.

Relatively few studies in Africa have explored beyond the role of knowledge and child-focused outcomes on EBF duration in the region, and even fewer studies have assessed the impact of outcome and normative beliefs on the duration of EBF [9-12]. Our objective was to determine the relationship between (i) knowledge and cessation of EBF, (ii) outcome expectancies and cessation of EBF, and (iii) normative beliefs and cessation of EBF among mothers in rural Kenya. We postulated that mother's knowledge of breastfeeding recommendations, mothers' expectations of the consequences of EBF on their child and on the mother, and mothers' perception of other people's support of EBF was significantly associated with EBF cessation in rural Kenya.

\section{Methods}

\section{Study design and study population}

The study utilized a cross-sectional design and was conducted in March-April 2013 in Kisumu West district in Kenya. The district is located on the shores of Lake Victoria in Nyanza province, a region with the highest prevalence of HIV in Kenya. HIV prevalence among adults $15-49$ years is $15 \%$ in Nyanza province compared to the country average of $7.4 \%$. The under-five mortality is the highest in Nyanza, with the rate of 149 deaths per 1000 live births.

The minimum number of the subjects 'n' required for survey was estimated at 335 using the formula $n=z^{2} p$ $(1-p) \div d^{2}$, where ' $z$ ' is the critical value and, in a twotailed test, is equal to 1.96 , 'p' is the estimated percent of Kenyan children under six months of age who are exclusively breast-fed at the time of the study, which, based on the Kenya Demographic Health Survey report, was taken as $32 \%$, and ' $d$ ' is the absolute sampling error that can be tolerated and was set at $5 \%$ [3]. A sample size of 400 was agreed upon to allow for approximately $20 \%$ attrition or incompletion rate.

The Community Health Workers (CHWs) in the study area keep up-to-date records of the number children less than five years old who reside within the area's 37 villages. A total of 9653 children 0-24 months old were identified from the CHWs records. The study sample was composed of children from all the villages with each village contributing proportionately, as defined by the number of children 0-24 months old. Thereafter, a convenience sampling procedure was utilized to identify study participants, in our case women of reproductive age with at least one child 0-24 months old and having resided in the study area for at least two years. Public health nurses, who had been trained to administer the questionnaires, were guided to potential study participants' homes by the CHWs. The household visits occurred between 9.00 am and $4.00 \mathrm{pm}$ on the weekdays. Potential study participants that lived closest to the main roads within each village and that were present at home during the visiting hours were approached and requested to participate in the study. All mothers who were approached agreed to participate in the study. Study participants' informed written consent was obtained prior to administering the questionnaires. The trained public health nurses administered the questionnaires. Because CHWs work with mothers on a daily basis, they were asked not to be present during the interviews to 
eliminate any influence their presence might have on the study participants' responses. The current research was conducted in accordance with the Helsinki Declaration and was approved by the Office of Research Subject Protections at George Mason University and the Kenya Medical Research Institute Ethical Review Committee. Hospital medical and nutrition officers were informed in detail about the aim and procedures of the study. Of the 400 mothers that participated in the study, 169 (42\%) had children less than 6 months old, 142 (36\%) had children between the ages of 6 and 12 months old and 89 (22\%) had children greater than 12 months old.

\section{Study questionnaire}

A questionnaire consisting of both closed and openended questions was developed to assess breastfeeding practices, EBF-related knowledge, outcome expectancies and social norms, and household socio-economic status and demographics as detailed below.

\section{Breastfeeding practices}

As part of the open-ended questions, mothers were asked to recall if the child was fed colostrum (locally referred to us "first milk"), the foods and drinks that the child consumed within the first three days of birth, how long after birth the child was introduced to breastmilk and to non-breastmilk foods and drinks including different types of water and medicines. Mothers were asked to indicate the different foods and drinks that the child had been introduced to by the time of the survey and to recall the age of the child at time of introduction to these foods and drinks. Mothers were also asked and to provide the reasons for introducing non-breastmilk foods to their children. A similar set of questions have been previously used in a similar population in Kenya [5]. Exclusive breastfeeding was defined as child receiving only breast-milk, and no other liquids or solids with the exception of medicinal drops or syrups.

\section{Knowledge, outcome expectancies and normative beliefs}

The questions on knowledge, outcome expectancies and normative beliefs were informed by questions and results from previous studies, including those conducted in sub-Sahara Africa [5, 9-14]. A total of 21 statements were developed to assess (i) knowledge of EBF-related recommendations (knowledge-7 statements), beliefs associated with impact on EBF on a child's health, growth and general well-being (outcome expectancies: child-5 statements), beliefs associated with impact on EBF on mothers health, physical appearance and ability to engage in other activities (outcome expectancies: mother5 statements), perception of acceptability of EBF by child's father, in-laws and community (normative beliefs- 3 statements) and whether the mother perceived breastfeeding in public to be embarrassing (1 statement). Mothers were asked to indicate if they agreed or disagreed (yes/no) with each statement.

\section{Household demographics and socioeconomic status}

Household members' birth dates, marital status, religion, tribe, sex, and number of completed school years were recorded. Household size and number of "under-5's" (children younger than 5 years old) within the household, maternal education level, and maternal age were defined from demographic data. Children's birth weight was collected from the maternal child health clinic cards. Additionally, mothers were asked to report on child's maturity status at birth. The socioeconomic status (SES) questionnaire, which has been used among populations in rural Kenya, accounted for salaried employment, income, land ownership and usage, education and literacy, household possessions and expenditures, types of houses, and involvement of parents in leadership and community positions $[5,15]$. Different weightings were assigned to household possessions depending on their value. A composite SES score was then developed, whereby a higher score represents a higher level of SES.

The questionnaire was prepared in the English language and translated into the local language, Dholuo. All questionnaires were pretested, and modified before data collection commenced.

\section{Data analysis}

Data analysis was performed using SAS version 9.1 (SAS Institute). Exclusive breastfeeding was defined as child receiving only breast milk and no other liquids or solids, with the exception of medicinal drops or syrups. EBF cessation occurred when a child consumed his/her first non-breastmilk food or drink (with the exception of medicinal drops or syrups). All psychosocial variables were first analyzed as individual indicators (reference categor$y=$ "disagree"). Composite scores were then created for knowledge and outcome expectancies and normative beliefs group of questions. Composite scores have been previously used in health-related research [16]. Responses to negatively-worded questions were recoded prior to creating the composite scores. Each correctlyanswered question or favorable response was assigned a score of 1 , which were added together to give a maximum score of 7 points for "knowledge", 5 points for "outcome expectancies: child", 5 points for "outcome expectancies: mother" and 3 points for EBF acceptability. The Cronbach's alpha coefficient was used to assess internal consistency reliability for the scores in each composite score. Cronbach's alpha coefficient values were 0.56 for "knowledge", 0.56 for "outcome expectancies: child", 0.71 for "outcome expectancies: mother" and 0.70 for EBF acceptability. "Knowledge" and "outcome 
expectancies: child" composite scores were dropped from further analyses due to the low Cronbach alpha coefficient values.

Cox regression analysis, a regression method used to investigate effect of various variables on time until event occurs, was used to assess the relationship between each independent variable of interest and EBF cessation, and the relationship between each composite score ("outcome expectancies: mother", "EBF acceptability") and EBF cessation. Finally, we ran a single multivariate model that included both "outcome expectancies: mother" and "EBF acceptability" composite scores. EBF cessation occurred when a child consumed his/her first non-breastmilk food or drink (with the exception of medicinal drops or syrups). The time variable was defined as the time from birth to the time of EBF cessation in months. An event was defined as "EBF cessation before 6 months". All observations that did not experience an event were right-censored. The regression analysis controlled for child's sex, mother's school years and the number of household members below five years of age. Proportionality assumption was assessed and met for all multivariate regression models.

\section{Results}

The mean age of the 400 mothers participating in the study was just above 27 years (Table 1). Only $3 \%$ of mothers attended post-secondary school and $1.8 \%$ of

Table 1 Study participants' socio-demographic characteristics ${ }^{a}$

\begin{tabular}{ll}
\hline & Estimate \\
\hline Mothers Characteristics & \\
Mothers' age (years) (mean \pm SD) & $27.3 \pm 7.2$ \\
School years (mean \pm SD) & $8.5 \pm 2.8$ \\
Highest grade: & \\
None & 1.8 \\
Some Primary & 27.9 \\
Completed Primary & 36.2 \\
Secondary School & 31.1 \\
Post-Secondary School & 3.0 \\
Children's Characteristics ${ }^{c}:$ & \\
Female & 49 \\
Age (months) (mean \pm SD) & $8.4 \pm 6.2$ \\
Less than 6 months old & 42.3 \\
6 to 12 months old & 35.5 \\
Greater than 12 months old & 22.3 \\
Birth weight (mean \pm SD) & $3.3 \pm 0.6$ \\
Low birth weight & 16 \\
Premature birth & 5.5 \\
\hline
\end{tabular}

${ }^{a}$ Estimates are percentages unless indicated otherwise

${ }^{\mathrm{b}} \mathrm{N}=399$ for mothers age and 398 for mother's education

${ }^{c} N=400$ for child's sex, age and prematurity status; $n=398$ for child's birth weight mothers had no schooling at all. The average age of the children in the study was approximately 8 months. Forty-two percent of the children, 0-24 months old, were under 6 months of age, $35.5 \%$ of children were 6 12 months of age and the remaining $22.3 \%$ were over 12 months of age.

Close to $100 \%$ study participants' breastfed at some point with $87 \%$ initiating breastfeeding within one hour of birth. Over $96 \%$ of the children had consumed colostrum within the first 3 days of life (Table 2). Over $89 \%$ of the mothers were breastfeeding at the time of the study. Over $68 \%$ of children less than 6 months old were exclusively breastfed at the time of the survey. A further assessment of children between the ages of 4 and 6 months showed that $55 \%$ of the children within this age-group were still exclusively breastfeeding at the time of the study.

Approximately $16 \%$ of the children had received nonbreastmilk liquids (non-medications) within the first 3 days of life, with gripe water being most-commonly consumed. Other non-breastmilk liquids/foods within the first three days of life included plain water, sugar or salt water mix and infant formula. A belief that breast milk was insufficient was the most common reason for introducing non-breastmilk foods. Less common reasons included the mother being sick, amongst others (Fig. 1). Unpasteurized cow's milk was the most common type of

Table 2 Breast feeding-related practices among mothers in rural Kenya $^{\mathrm{a}}$

\begin{tabular}{ll}
\hline & Percentage \\
\hline Ever breast fed & 99.8 \\
Timely breast feeding initiation $^{\text {b }}$ & 87.5 \\
Within first 3 days of life ${ }^{\text {b: }}$ & \\
Colostrum & 96.7 \\
Medication & 14.3 \\
Gripe water & 8.8 \\
Plain water & 3.8 \\
Sugar or salt water & 2.8 \\
Formula & 2.3 \\
Breast feeding at time of study: & \\
All children & 89.2 \\
Less than 6 months old & 98.8 \\
6 to 12 months old & 92.9 \\
Greater than 12 months old & 65.2 \\
Exclusive breastfeeding at time of study: & \\
Less than 6 months old & 68.0 \\
4 to $<6$ months old & 55.3 \\
6 to 12 months old & 7.8 \\
Greater than 12 months old & 0.0 \\
\hline
\end{tabular}

${ }^{\mathrm{a}} \mathrm{N}=400$ unless indicated otherwise

${ }^{\mathrm{b}} \mathrm{N}=399$ 


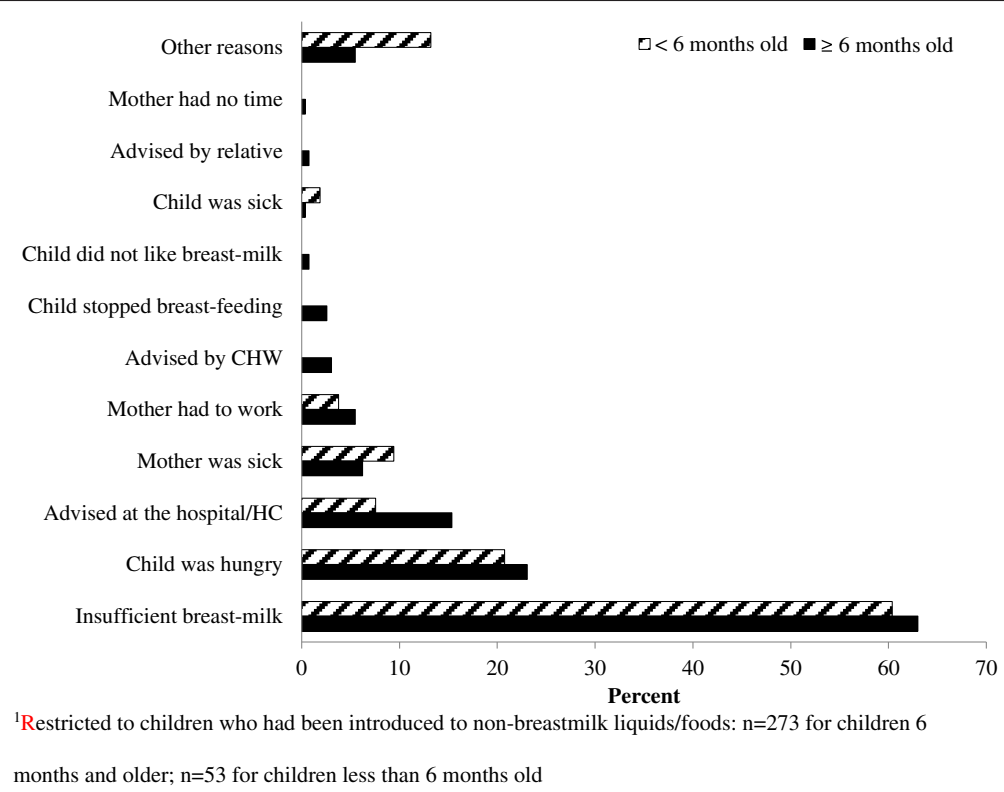

Fig. 1 shows the reasons, given by mothers, for introducing non-breast milk liquids/food to young children with "insufficient breast milk" and "child being hungry" as the most common reasons

non-breast milk consumed by the children at the time of the study (Fig. 2).

Psychosocial factors and premature cessation of exclusive breastfeeding

More than $80 \%$ of the mothers correctly identified the EBF-related recommendations (Table 3). Five of the seven knowledge variables were significantly associated with premature EBF cessation. Mothers who agreed with the following statements "a new-born child should be fed only on breast-milk" and "a child less than 6 months old should be fed only on breast-milk" were associated with significantly lower risks of premature EBF cessation (Table 3). Mothers who agreed with the statements "it is important to give a new-born child other liquids like water", "it is important to give a new-born child other

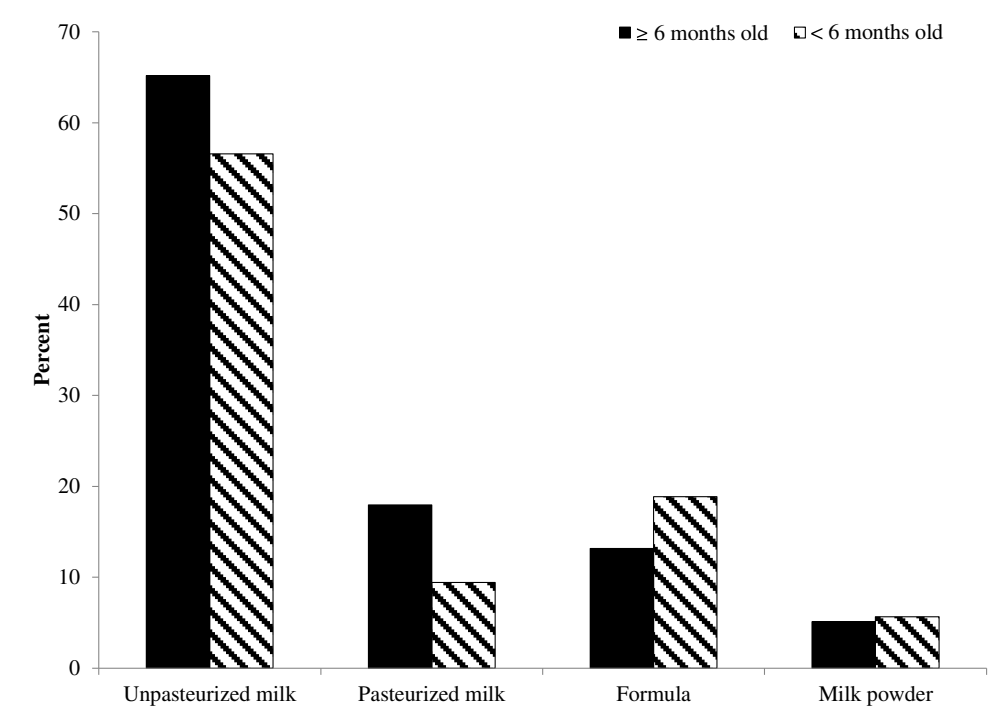

${ }^{1}$ Restricted to children who had been introduced to non-breastmilk liquids/foods: $n=273$ for children 6

months and older; $\mathrm{n}=53$ for children less than 6 months old

Fig. 2 shows that unpasteurized cow's milk is the most common breast milk substitute in rural 
Table 3 Predicting premature exclusive breast feeding cessation among mothers in rural Kenya ${ }^{a}$ b

\begin{tabular}{|c|c|c|c|c|c|}
\hline Variable & Percent/mean score & SD & Median & $\mathrm{HR}$ & $95 \% \mathrm{Cl}$ \\
\hline \multicolumn{6}{|l|}{ Breastfeeding-related knowledge } \\
\hline A child should be breast-fed within one hour of his/her birth (\%) & 96 & - & - & 0.70 & $0.30,1.65$ \\
\hline A new-born child should be fed only on breast-milk (\%) & 96 & - & - & $0.42^{*}$ & $0.18,0.97$ \\
\hline It is important to give a new-born child other liquids like water (salty, sugary or plain) & 19 & - & - & $2.52^{*}$ & $1.68,3.80$ \\
\hline It is important to give a new-born child other foods like porridge, tea, juice, etc. & 12 & - & - & $1.86^{*}$ & $1.13,3.04$ \\
\hline A child less than 6 months old should be fed only on breast-milk (no water) & 84 & - & - & $0.37^{*}$ & $0.24,0.57$ \\
\hline A child who is less than 6 months old should be breastfed on demand & 90 & - & - & 0.87 & $0.48,1.55$ \\
\hline It is important to give other liquids/foods to a child before he/she reaches 6 months old & 12 & - & - & $2.40^{*}$ & $1.51,3.83$ \\
\hline \multicolumn{6}{|l|}{ Outcome expectancies: child } \\
\hline \multicolumn{6}{|l|}{ A child less than 6 months old who is fed only breastmilk will: } \\
\hline get hungry more quickly compared to a child who is fed other foods/drinks & 34.50 & - & - & $1.62^{*}$ & $1.09,2.39$ \\
\hline gain less weight compared to a child who is fed other foods/drinks & 26.25 & - & - & $1.80^{*}$ & $1.20,2.70$ \\
\hline have fewer diseases (like diarrhea) compared to a child who is fed other foods/drinks & 72.50 & - & - & 1.48 & $0.92,2.38$ \\
\hline have fewer stomach problems compared to a child who is fed other foods/drinks & 72.75 & - & - & 1.08 & $0.68,1.70$ \\
\hline feel more thirsty compared to a child who is fed other foods/drinks & 21.25 & - & - & $1.78^{*}$ & $1.17,2.70$ \\
\hline \multicolumn{6}{|l|}{ Outcome expectancies: mother } \\
\hline \multicolumn{6}{|l|}{ A mother who feeds her young child, less than 6 months old, only breast-milk: } \\
\hline will look thinner compared than a mother who feeds her young child other foods/drinks & 21.25 & - & - & $2.43^{*}$ & $1.62,3.66$ \\
\hline $\begin{array}{l}\text { will feel hungry more quickly compared to a mother who feeds her young child } \\
\text { other foods/drinks }\end{array}$ & 38.50 & - & - & $2.31^{*}$ & $1.55,3.42$ \\
\hline $\begin{array}{l}\text { will get sick/ill more easily compared to a mother who feeds her young child other } \\
\text { foods/drinks }\end{array}$ & 13.25 & - & - & $2.70^{*}$ & $1.73,4.21$ \\
\hline $\begin{array}{l}\text { will have more sagging breasts compared to a mother who feeds her young child } \\
\text { other foods/drinks }\end{array}$ & 29.75 & - & - & $2.13^{*}$ & $1.44,3.16$ \\
\hline $\begin{array}{l}\text { cannot do other things outside the home or go places compared to a mother who } \\
\text { feeds her young child other foods/drinks }\end{array}$ & 20.00 & - & - & $2.37^{*}$ & $1.57,3.59$ \\
\hline "Outcome expectancies: mother" score & 3.77 & 1.44 & 4.00 & $0.70^{*}$ & $0.62,0.79$ \\
\hline \multicolumn{6}{|l|}{ Normative beliefs } \\
\hline EBF not acceptable to child's father (\%) & 14 & - & - & $2.17^{*}$ & $1.38,3.42$ \\
\hline EBF not acceptable to in-laws (\%) & 23 & - & - & $2.02^{*}$ & $1.34,3.04$ \\
\hline EBF not acceptable in community (\%) & 18 & - & - & 1.50 & $0.95,2.35$ \\
\hline EBF acceptability score & 2.44 & 0.92 & 3.00 & $0.72^{*}$ & $0.60,0.86$ \\
\hline It is embarrassing to breast feed in public (\%) & 24 & - & - & $1.81^{*}$ & $1.21,2.73$ \\
\hline
\end{tabular}

pvalue $<0.05$

$C l$ confidence interval, EBF exclusive breast feeding, $H R$ hazards ratio, $S D$ standard deviation

$\mathrm{HR}$ and associated $\mathrm{Cl}$ from each row represents results from one multiple survival analysis regression model that includes child's sex, mother's school years and

the number of household members below five years of age

${ }^{\mathrm{a}} \mathrm{N}=394$

${ }^{\text {b}}$ Reference category for all non-composite score variables="disagree"

foods like porridge, tea, juice, etc." and "it is important to give other liquids/foods to a child before he/she reaches 6 months old" were associated with significantly higher risks of premature EBF cessation.

Over $65 \%$ of the mothers selected positive responses to statements expressing impact of EBF on the child. Mothers who indicated that a young child (less than 6 months old) who was fed only breastmilk would feel hungry more quickly, gain less weight and feel thirstier were associated with significantly higher risk of premature EBF cessation. Over $61 \%$ of the mothers selected positive responses to statements expressing impact of EBF on the mother. Mothers who indicated that a mother who fed only breastmilk to her young child (less than 6 months old) would be thinner, feel hungry more quickly, get sick more easily, have more sagging breasts and cannot do other things outside the home or go places were associated with significantly higher risk of premature 
EBF cessation. "Outcome expectancies: mother" scores averaged 3.77 (out of 5) with a median of 4 points. A oneunit increase in positive perception of the impact of EBF on the mother's health, appearance and activities was associated $30 \%$ lower risk of premature EBF cessation.

Over 85, 76 and $81 \%$ of the mothers perceived EBF to be acceptable to the child's father, their in-laws and in their community, respectively. Mothers who indicated that EBF was acceptable to the child's father and to their inlaws were associated with significantly lower risk of premature EBF cessation. The overall EBF acceptability score averaged at 2.44 (out of 3). A one-unit increase in EBF acceptability score was associated $28 \%$ lower risk of premature EBF cessation. Over $24 \%$ of the mothers perceived breastfeeding in public to be embarrassing. Mothers who perceived breastfeeding in public to be embarrassing, compared to those who did not, were associated with $81 \%$ higher risk of premature EBF cessation (Table 3).

Results from the final regression model showed that having a more positive perception of the impact of EBF on mother's health and appearance and an increase in EBF acceptability were each associated with significantly lower risk [(HR: 0.71; CI: 0.63-0.80) and (HR: 0.76; CI: 0.63-0.91), respectively] of premature EBF cessation. When we limited the analysis to mothers with children of ages 6 months and above $(n=231)$, the results showed that having a more positive perception of the impact of EBF on mother's health and appearance and an increase in EBF acceptability were each associated with significantly lower risk [(HR: 0.73; CI: 0.62-0.86) and (HR: 0.77; CI: 0.59-0.99), respectively] of premature EBF cessation.

\section{Discussion}

Over $99 \%$ of the mothers included in the current study initiated breastfeeding. Timely initiation of breastfeeding was considerably higher than the national average [3]. Over $68 \%$ of children less than 6 months old and $55 \%$ of children within the 4-5 month age-group were exclusively breastfed at the time of the study. Both rates were slightly higher than the national rates. According to results from the DHS conducted in 2014, $61 \%$ of children less than 6 months old and $45 \%$ of children within the 4-5 month age-group were exclusively breastfed in Kenya [6].

Study participants beliefs or actions were in agreement with the EBF-related recommendations for the most part. About $96 \%$ of the mothers agreed that a newborn child should be fed only breastmilk, $84 \%$ initiated breastfeeding in a timely manner and $96 \%$ of the children had consumed colostrum within the first three days of life. However, our results also revealed certain instances of lack on concurrence in mothers' beliefs. For example, while nearly all mothers agreed with the statement that a newborn child should be fed only breastmilk, close to $20 \%$ of the mothers believed that it was important to give other liquids to a newborn child. Such a disconnect might be a reflection of the differences between health care workers' recommendations and one's cultural or social norms and expectations. While breastfeeding initiation is expected within most communities in Africa, EBF may not be culturally expected or encouraged $[5,10,13,17]$. We believe that EBF promotion efforts might become more effective if there is a better understanding of the reasons behind the differing or contrasting beliefs and appropriate efforts made to address any inconsistencies. Mother's knowledge levels were high and this was similar to what has been reported in other studies in Africa $[18,19]$. Five of the seven knowledge items included in the current study were each significantly associated with premature EBF cessation. In addition, increase in overall knowledge of the EBF-related recommendations was shown to be protective of EBF.

Mothers were slightly less enthusiastic when it came to child-related outcome expectancies. Only $72 \%$ of the mothers believed that an exclusively breastfed child experienced less illness and stomach problems compared to those who were not exclusively breastfed. However, it is important to note that the reported $72 \%$ is higher than what was noted in other rural communities in Kenya [10]. Over $34 \%$ of the mothers believed that exclusively breastfed children became hungry more quickly while over $20 \%$ believed exclusively breastfed children became thirstier and gained less weight compared to those who were not exclusively breastfed. Each of these three variables, in addition to the overall "outcome expectancies: child" score were significantly associated with premature EBF cessation. Child crying, child being hungry or child not gaining adequate weight have been previously cited as reasons for mothers choosing not to practice EBF in Africa [5, 14, 19]. For this group of mothers, mother's perception of the impact of EBF on occurrence of childhood illnesses was not significantly associated with premature EBF cessation. However, perceived differences in children's satiety and rate of growth were important determinants of EBF cessation in this group of mothers. While breastfeeding promotion messages have continuously addressed the benefits of breastfeeding, these messages may not have directly addressed differences in children's satiety or growth patterns associated with the different child feeding patterns.

Low levels of enthusiasm were also noted in mothers' perception of the impact of EBF on the mother's health, physical appearance and ability to engage in other activities. Over $38 \%$ of the mothers believed that mothers who practiced EBF became hungry more quickly compared to mothers who did not practice EBF. And the current study's results show that these mothers were associated with $131 \%$ higher risk of stopping EBF before six months post-partum. Mothers have previously 
indicated that their food security/nutritional status was an important determinant of breastfeeding practices [5]. Mothers' physical appearance was also noted to be important with 20-30 \% of the mothers indicating that mothers who practiced EBF looked thinner or had more sagging breasts. This is an important factor, considering that there is a general preference for a curvier and fuller body amongst African populations and being thin is associated with poor health [20-22]. Each of the "outcome expectancies: mother" variables in addition to the composite score were significantly associated with premature EBF cessation. Study participants who believed that mothers who practiced EBF looked thinner and those that believed that mothers who practiced EBF had more sagging breasts were associated with $143 \%$ and $131 \%$ higher risk of stopping EBF before six months postpartum. To our knowledge, this is one of very few studies that have explored mother's perception of the impact of EBF on mothers' health, physical appearance and ability to engage in other activities. Over $71 \%$ of mothers in Kenya have previously reported that breastfeeding was a time-wasting activity that reduced time for recreational activities, while only $42 \%$ of mothers interviewed in Nigeria indicated that breastfeeding had an effect on care of other family members and on marital relationship $[10,11]$. The current study adds to the "time-demand" literature and introduces the aspect of mothers' health and physical appearance as important topics that need to be addressed when promoting EBF. It is important to note that mothers may not feel comfortable raising any non-health related concerns with healthcare workers. Results from the current study indicate that these issues need to be addressed so as to identify relevant strategies aimed at improving EBF rates in the country.

Over $80 \%$ of the mothers perceived EBF to be acceptable to the child's father and in their community. A slightly lower percent (76\%) perceived EBF to be acceptable to their in-laws. Mothers' perception of the acceptability EBF to the child's father or to their in-laws in addition and the overall EBF acceptability score were significantly associated with EBF cessation, thus highlighting the importance of normative beliefs in determining breastfeeding-related practices for this group of mothers. Previous studies in Africa have reported the possible influence of partners and in-laws on breastfeeding practices $[5,11]$. Injunctive normative beliefs were shown to be a significant determinant of mothers' intention to practice EBF in Malaysia [23]. Our findings indicate that mothers' beliefs or perceptions on how important others and their community view EBF is an important factor in their decision-making process and lends support to efforts aimed at including male partners and in-laws in breastfeeding promotion [24]. Study participants who believed that EBF was not acceptable to the child's father and those that believed that EBF was not acceptable to their in-laws were associated with a $117 \%$ and $102 \%$ higher risk of stopping EBF before six months post-partum, respectively while those who indicated that it was embarrassing to breastfeed in public were associated with $81 \%$ higher risk of stopping EBF before six months post-partum.

The current study's limitation lies in the potential bias associated with the selection of the study participants. The retrospective recall of breastfeeding practices presents another set of potential bias which may include reporting of desired practices and error in times of introduction of particular foods. It is also important to note that the study was conducted in only one district in Kenya and the results should not be generalized across the whole country. While acknowledging that a prospective longitudinal study and a random selection of study participants would be better alternatives, the use of a cross-sectional study design and a convenience sample was most feasible with the available resources. Multiple strategies were taken to help minimize potential recall bias. These included the use of health workers who were familiar with the study population, selection of study participants from all 37 villages found in the study area, requiring CHWs not to be present when questionnaires were administered, rigorous training and standardization of study procedures, field-based supervision, and regular meetings to continually assess progress. Another study limitation may lie in the use of "knowledge", "outcome expectancies" and "EBF acceptability" questions that have not been validated for use in the study population. We selected questions based on what was utilized or reported in previous studies including those conducted in sub-Sahara Africa. However, we would recommend that the questions' validity should be assessed in Kenya and similar populations. The strengths of this study lies in its analytical nature that goes beyond describing factors to assessing the extent to which specific psychosocial factors influence EBF cessation within a rural setting in Africa. The use of the Cox Proportional Model accounts for the individual differences in time between birth and EBF cessation and also accounts for individuals who do not experience EBF cessation within the first six months post-partum. In addition, the study addressed certain factors that have not been previously examined as possible determinants of EBF in sub-Sahara Africa. Our analysis did not utilize the "knowledge" and "outcome expectancies: child" composite scores due to the low Cronbach values and we recommend additional examination of these composite score constituents prior to using them in further research $[25,26]$.

\section{Conclusion}

The study adds to the existing literature by going beyond knowledge and child-related beliefs to include beliefs 
about the impact of EBF on the mother and EBF-related normative beliefs in its assessment. Mother's perceptions of the impact of EBF on the mother's health, physical appearance and ability to engage in other activities were shown to consistently have the strongest relationship with premature EBF cessation in this group of mothers. Addressing these beliefs has the potential to contribute to more effective EBF promotion efforts in rural Kenya.

\section{Availability of data and materials}

The dataset supporting the conclusions of this article is available in the George Mason University Dataverse repository in http://arc.irss.unc.edu/dvn/dv/gmu.

\section{Abbreviations}

CHWs: Community Health Workers; EBF: Exclusive Breastfeeding; HIV: Human immunodeficiency virus; MCH: Maternal-and-child health; WHO: World Health Organization.

\section{Competing interests}

The authors declare that they have no competing interests.

\section{Authors' contribution}

CAG conceived the study, designed and conducted the research, analyzed the data and wrote the paper. JC conceived the study, facilitated the field activities, conducted the research and edited the paper. Both authors read and approved the final manuscript.

\section{Acknowledgement}

We are grateful to Heibatollah Baghi for providing guidance on the statistical analysis.

Preliminary results of these analyses were presented at Federation of American Societies for Experimental Biology (FASEB) conference, Boston, USA. 2015. Presentation title: Psychosocial determinants of exclusive breastfeeding in rural Kenya.

\section{Funding source}

Financial support for the research, authorship, and/or publication of this article received from George Mason University Research Seed Grant Award. No funding body had any role in the study design, data collection and analysis, decision to publish, or preparation of the manuscript.

\section{Author details}

'Department of Nutrition \& Food Studies, College of Health \& Human Services, George Mason University, 4400 University Dr. MSN 1 F8, Fairfax, VA 22030, USA. ${ }^{2}$ Ministry of Health, Kisumu County, Kenya.

Received: 17 September 2015 Accepted: 22 February 2016 Published online: 09 March 2016

\section{References}

1. World Health Organization (WHO). HIV and Infant Feeding: New Evidence and Programmatic Experience: Report of a Technical Consultation Held on Behalf of the Inter-Agency Task Team (IATT) on Prevention of HIV Infections in Pregnant Women, Mothers and Their Infants. Geneva: World Health Organization (WHO); 2007.

2. World Health Organization (WHO). HIV and Infant Feeding: Principles and Recommendations for Infant Feeding in the Context of HIV and Summary of Evidence. Geneva: World Health Organization (WHO); 2010.

3. Kenya National Bureau of Statistics (KNBS). MEASURE DHS. Kenya: DHS 20082009 final report.

4. Kimani-Murage EW, Kyobutungi C, Ezeh AC, Wekesah F, Wanjohi M, Muriuki P. Musoke RN, Norris SA, Griffiths P. Madise NJ. Effectiveness of personalised, home-based nutritional counselling on infant feeding practices, morbidity and nutritional outcomes among infants in Nairobi slums: study protocol for a cluster randomised controlled trial. Trials. 2013;14:445.
5. Gewa CA, Oguttu M, Savaglio L. Determinants of early child-feeding practices among HIV-infected and noninfected mothers in rural Kenya. J Hum Lact. 2011;27:239-49.

6. Kenya National Bureau of Statistics (KNBS), Ministry of Health (MOH), National AIDS Control Council (NACC), Kenya Medical Research Institute (KEMRI), National Council for Population and Development. Kenya: DHS 2014 key indicators report.

7. Ministry of Health (MOH), World Health Organization (WHO), UNICEF. National Strategy for Maternal and Infant and Young Child Nutrition 2012-2017.

8. Contento IR. Nutrition Education: Linking Research, Theory, and Practice. 2nd ed. Sudbury: Jones and Bartlett Learning; 2011.

9. Agunbiade OM, Ogunleye OV. Constraints to exclusive breastfeeding practice among breastfeeding mothers in Southwest Nigeria: implications for scaling up. Int Breastfeed J. 2012;7:5.

10. Walingo M, Mutuli L. Influence of maternal beliefs, attitude, perceived behavior on breast-feeding among post partum mothers in western Kenya. PJN. 2014;13:250-4.

11. Mbada CE, Olowookere AE, Faronbi JO, Oyinlola-Aromolaran FC, Faremi FA, Ogundele AO, Awotidebe TO, Ojo AA, Augustine OA. Knowledge, attitude and techniques of breastfeeding among Nigerian mothers from a semiurban community. BMC Res Notes. 2013;6:552.

12. Aubel J. The Role and Influence of Grandmothers and Men: Evidence Supporting a Family-Focused Approach to Optimal Infant and Young Child Nutrition. Washington, DC: Infant and Young Child Nutrition Project/PATH; 2011.

13. Leshabari SC, Blystad A, Moland KM. Difficult choices: Infant feeding experiences of HIV-positive mothers in northern Tanzania. SAHARA J. 2007;4:544-55.

14. Cherop CE, Keverenge-Ettyang AG, Mbagaya A. Barriers to exclusive breastfeeding among infants and 0-6 months in Eldoret Municipality in Kenya. East Afr J Public Health. 2009;6:69-72.

15. Neumann CG, Bwibo NO, Murphy SP, Sigman M, Whaley S, Allen LH, Guthrie D, Weiss RE, Demment MW. Animal source foods improve dietary quality, micronutrient status, growth and cognitive function in Kenyan school children: background, study design and baseline findings. J Nutr. 2003;133(11 Suppl 2):3941S-9S

16. Baghi H, Atherton M. Construct validity and reliability of scores on scales to measure the impairment of health-related quality of life in persons with asthma. J Nurs Meas. 2004;12:21-31.

17. Sibeko L. Beliefs, attitudes, and practices of breastfeeding mothers from a periurban community in South Africa. J Hum Lact. 2005;21:31-8.

18. Katepa-Bwalya M, Mukonka V, Kankasa C, Masaninga F, Babaniyi O, Siziya S. Infants and young children feeding practices and nutritional status in two districts of Zambia. Int Breastfeed J. 2015;10:5.

19. Onah S, Osuorah DI, Ebenebe J, Ezechukwu C, Ekwochi U, Ndukwu I. Infant feeding practices and maternal socio-demographic factors that influence practice of exclusive breastfeeding among mothers in Nnewi South-East Nigeria: a cross-sectional and analytical study. Int Breastfeed J. 2014;9:6.

20. Mvo Z, Dick J, Steyn K. Perceptions of overweight African women about acceptable body size of women and children. Curationis. 1999;22:27-31.

21. Holdsworth M, Gartner A, Landais E, Maire B, Delpeuch F. Perceptions of healthy and desirable body size in urban Senegalese women. Int J Obes. 2004:28:1561-8.

22. Puoane T, Tsolekile L, Steyn N. Perceptions about body image and sizes among Black African girls living in Cape Town. Ethn Dis. 2010;20:29-34.

23. Ismail TAT, Muda WMW, Bakar MI. Intention of pregnant women to exclusively breastfeed their infants: the role of beliefs in the theory of planned behaviour. J Child Health Care. 2014;18:123-32.

24. Aubel J. The Roles and Influence of Grandmothers and Men: Evidence Supporting a Family-Focused Approach to Optimal Infant and Young Child Nutrition. 2011: PATH; [Infant and Young Child Nutrition Project]

25. Bland JM, Altman DG. Statistics notes: Cronbach's alpha. BMJ. 1997;314:572.

26. George D, Mallery P. SPSS for Windows Step by Step: A Simple Guide and Reference, 11.0 Update. Boston: Allyn and Bacon; 2003. 